\title{
The SMarT Classifier for Arabic Fine-Grained Dialect Identification
}

\author{
Karima Meftouh \\ Badji Mokhtar University \\ Annaba - Algeria \\ karima.meftouh@univ-annaba.dz
}

Salima Harrat

École Normale Supérieure de Bouzaréah

Algiers - Algeria

slmhrrtegmail.com

\author{
Karima Abidi \\ Loria - Univ. Lorraine \\ Nancy - France \\ karima.abidieloria.fr
}

\author{
Kamel Smaïli \\ Loria - Univ. Lorraine \\ Nancy - France \\ kamel.smailieloria.fr
}

\begin{abstract}
This paper describes the approach adopted by the SMarT research group to build a dialect identification system in the framework of the Madar shared task on Arabic fine-grained dialect identification. We experimented several approaches, but we finally decided to use a Multinomial Naïve Bayes classifier based on word and character ngrams in addition to the language model probabilities. We achieved a score of $67.73 \%$ in terms of Macro accuracy and a macro-averaged F1-score of $67.31 \%$.
\end{abstract}

\section{Introduction}

Arabic is a complex language which presents significant challenges for natural language processing and its applications. Arabic is characterized by its plurality. It consists of a wide variety of languages, which includes the Modern Standard Arabic (MSA), and a set of various dialects differing according to regions and countries.

Language identification is the task of identifying the language of a given text. It is an important preprocessing step for many Natural Language Processing (NLP) tasks such as machine translation (Meftouh et al., 2018; Harrat et al., 2017), sentiment analysis (Rana et al., 2016; Abdul-Mageed et al., 2014; Saad et al., 2013), etc. In general, language identification is not a high challenging issue since this research has been supported for a long time and several machine learning techniques have been tested in this area that yielded to more or less good results. Nonetheless, in cases such as identifying languages from very little data, from mixed input or when the languages are extremely close to each other, the task becomes very challenging (Goutte et al., 2014).

This paper describes the submission of Loria (SMarT research group) to the Madar shared task on Arabic fine-grained dialect identification covering 25 specific cities from across the Arab World, in addition to Modern Standard Arabic (Bouamor et al., 2019). This shared task is the first to target a large set of dialect labels at the city and country levels. It has two subtasks.

Subtask 1: MADAR Travel Domain Dialect Identification.

Subtask 2: MADAR Twitter User Dialect Identification.

Our submission to this campaign is dealing with the first subtask.

The remainder of this paper is organized as follows: in the next section, we discuss related work pertaining to Arabic dialect identification. Section 3 reviews the modeling choices we made for the shared task, and Section 4 describes results in detail.

\section{Related Work}

Several research works addressed the problem of Arabic dialect identification. The authors of Habash et al. (2008) presented standard annotation guidelines to identify the switching between the MSA and at least one dialect. These guidelines can be used to annotate large collections of data used for training and testing NLP tools. In Zaidan and Callison-Burch (2012), a large annotated dataset, created by harvesting an important number of reader commentaries on online newspapers content, is used to train and evaluate automatic classifiers for dialect detection and identification. The authors crowdsourced an annotation task to obtain sentence-level labels indicating what proportion of the sentence is dialectal, and which dialect the sentence is written in. The approach used in dialect identification relies on training language models for the different varieties of Arabic. Another work presents a supervised approach for performing sentence level dialect identification 
between Modern Standard Arabic and Egyptian Dialectal Arabic (Elfardy and Diab, 2013). The authors use token level labels to derive sentencelevel features. These features are then used with other core and meta features to train a generative classifier that predicts the correct label for each sentence in the given input text. In addition to a multi-dialect, multi-genre, human annotated corpus, the authors in Cotterell and Callison-Bursh (2014) present the results of a language identification task extended to include 5 dialects. They considered Naïve Bayes and Support Vector Machines. The approach used by Darwish et al. (2014) for the identification of the Egyptian dialect was based on lexical, morphological and phonological information. They show that accounting for such information can improve dialect detection accuracy by nearly $10 \%$. Using a set of surface features based on characters and words Malmasi et al. (2015) conduct three experiments with a linear SVM classifier and a meta-classifier using stacked generalization on the Multidialectal Parallel Corpus of Arabic (MPCA) compiled by Bouamor et al. (2014). They first conduct a 6way multi-dialect classification task then investigate pairwise binary dialect classification and finally conduct cross-corpus evaluation on the Arabic Online Commentary (AOC) dataset. In AlBadrashiny et al. (2015), the authors present a hybrid approach for performing token and sentence levels Arabic Dialect Identification. The token level component relies on a Conditional Random Field (CRF) classifier that take decisions based on several underlying components such as language models, a named entity recognizer and a morphological analyzer to label each word in the sentence. The sentence level component uses an ensemble of classifiers that models different aspects of the language. In another work, Al-Badrashiny and Diab (2016) present a system that detects points of code-switching in sentences between the MSA and dialectal Arabic. In Sadat et al. (2014), the authors present a bi-gram character-level model to identify the dialect of sentences, in the social media context, among dialects of 18 Arab countries. Bougrine et al. (2015) addressed the problem of spoken Algerian dialect identification by using prosodic speech information (intonation and rhythm). They performed an experiment on six dialects from different Algerian regions. In Salameh et al. (2018), the authors present the first system dealing with fine-grained dialect classification task and covering 25 specific cities from across the Arab World, in addition to Standard Arabic. For this purpose, they build several classification systems using a Multinomial Naïve Bayes classifier and exploring a large space of features.

\section{The Modeling Choices}

\subsection{Data}

For the experiments reported in this paper, we only use the training and the development data available in the subtask 1 of the shared task. The dataset of this subtask is the same as the one reported on Bouamor et al. (2018) and Salameh et al. (2018). It is composed of two corpora. The first (Corpus-26) is a collection of parallel sentences, built to cover the dialects of 25 cities from the Arab World, in addition to MSA. The training part consists of 1600 labeled instances per class, while the development part has 200 labeled instances per class. The second (Corpus-6) contains 10, 000 additional sentences translated to the dialects of only five cities: Beirut, Cairo, Doha, Tunis and Rabat, in addition to MSA. They are splitted on two categories: 9,000 instances per language for the training and 1,000 instances per language for the development.

\subsection{Method}

In order to develop a language identification system that can distinguish between several Arabic dialects, we tested three methods namely simple neural networks (LSTM) (Sak et al., 2015), a method based on word embedding (Word2vec) (Mikolov et al., 2013) and Naïve Bayes classifiers. Given the limited size of the provided corpora, the first two methods have proven ineffective. We give in Table ?? the results we obtain using Corpus 26 in terms of Macro averaged F1-score, precision and recall.

\begin{tabular}{|l|l|l|l|}
\hline \multirow{2}{*}{ Method } & \multicolumn{3}{|c|}{ Corpus 26 } \\
\cline { 2 - 4 } & Precision & Recall & F1-score \\
\hline Word2vec & 50.11 & 49.90 & 49.74 \\
\hline LSTM & 58.04 & 61.54 & 58.33 \\
\hline
\end{tabular}

Table 1: Macro averaged F1-score, Precison and Recall for Word2vec and LSTM method.

We used a Naïve Bayes method because in the past, we did a comparative study of methods for Topic identification. This method for French leads 
to the best results (Bigi et al., 2001). In this work we consider a Multinomial Naïve Bayes classifier, in fact a study proposed in McCallum and Nigam (1998) showed that the multinomial model is found to be almost better than the multivariate Bernoulli model and the experimental results yielded to better results. So, we consider a Multinomial Naïve Bayes classifier for this task. In this case, the term Multinomial Naïve Bayes lets us know that each $p\left(f_{i} \mid c\right)$ (where $f_{i}$ is a feature and $c$ the category or the class) is a multinomial distribution, rather than some other distribution such as a Bernoulli distribution.

To develop our system, we used Python, relying on Scikit-Learn module (Pedregosa et al., 2011).

\subsection{Features}

A Naïve Bayes model classifier identifies a category by calculating the distributions of the features within a category. It also assumes that each of the features it uses are conditionally independent of one another given a category. Identifying features is a critical step when applying Naïve Bayes classifiers. That is why we did several experiments to select some adequate features. After several experiments, we selected for each sentence, the following 38 features as follows:

- A unigram of words.

- A bigram of words

- Character n-grams: from 1 to 5

- Character n-grams: from 1 to 5 , by taking into account the spaces between words; in other words ngrams at the edges of words are padded with space. All the symbols of punctuation have been removed from the training, development and test data.

- 26 likelihoods estimated by the 26 unigram language models

For all the features, we use a special character to mark the start of the sentences. We utilize Term Frequency-Inverse Document Frequency (Tf-Idf) scores (Spärck Jones, 1972) as it has been shown to outperform count weights in several NLP applications.

\section{Results and Discussion}

For the purpose of this campaign, we built several systems using the model described in section
3. We did several experiments to determine the smoothing adding value, necessary for the Naive Bayes method, and we set it to 0.093 for all the systems. In Table 2, we report the results of all the experiments concerning the Multinomial Naive Bayes method. For the evaluation purpose, we use the Macro averaged F1-score which is retained as the official metric by the organizers of Madar shared task.

\begin{tabular}{|c|lc|c|c|}
\hline \multicolumn{3}{|c|}{ Ngrams features } & \multicolumn{2}{c|}{ F1-score } \\
\hline Word & Char_wo & Char_wi & Dev & Test \\
\hline 1 & - & - & 63.03 & 62.31 \\
$1-2$ & - & - & 63.27 & 62.32 \\
$1-3$ & - & - & 63.04 & 61.96 \\
\hline \hline- & $1-3$ & - & 59.28 & 57.25 \\
- & $1-4$ & - & 64.50 & 63.99 \\
- & $1-5$ & - & 66.27 & 65.33 \\
\hline \hline- & - & $1-3$ & 59.66 & 57.62 \\
- & - & $1-4$ & 64.45 & 63.21 \\
- & - & $1-5$ & 66.50 & 64.40 \\
\hline \hline- & $1-5$ & $1-5$ & 66.92 & 65.56 \\
$1-2$ & $1-5$ & $1-5$ & 69.06 & 67.34 \\
$1-2$ & $1-5$ & $1-5$ & 69.09 & 67.31 \\
& \multicolumn{4}{|c}{} \\
\hline
\end{tabular}

Table 2: Macro averaged F1-score on Development and Test sets for Corpus-26.

First, we train the multinomial NB on word ngrams. The best results are achieved with the use of unigrams and bigrams. For higher order of $\mathrm{n}$-grams, the performance of the model degrades due to the data sparsity. Then, we tested the effect of character ngrams features with (wi) and without (wo) taking into account the space at the end of the words. We experimented using the features of each option alone and combined. In Table 2 the symbol $x-y$ means that all the $\mathrm{n}$-grams features from $x$ to $y$ of the corresponding column are taken into account in the classification.

In all the experiments, the best model is obtained for $\mathrm{n}$ ranging from 1 to 5 . We remark that a classifier based on character ngrams features (1-5) outperforms the classifier based on word ngrams features by at least 3 points. Finally, the best classifier is the one using word unigrams and bigrams, and character ngrams ranging from 1 to 5 with and without space. The introduction of the language model features improved the result on the development corpus and reduced it on the test corpus. We decided finally to participate to the campaign 
with the classifier including the language model parameters.

\section{Conclusion}

In this paper, we described the experiments we conducted as part of the MADAR shared task on Arabic fine-grained dialect identification. This task is the first covering the dialects of 25 specific cities from across the Arab World, in addition to MSA. Thus, we tested several systems exploring a large set of features. A blind run on the test set was then performed and submitted as part of the shared task. The Macro accuracy is $67.73 \%$ (macro-averaged F1-score $67.31 \%$ ), placing our classifier first among 19 participants. This result shows that our approach despite its simplicity performs very well and even if it is ranked first, we need to make more efforts to make it powerful so that it can become an effective tool for the community.

\section{References}

Muhammad Abdul-Mageed, Mona Diab, and Sandra Kbler. 2014. SAMAR: Subjectivity and sentiment analysis for Arabic social media. Computer Speech \& Language, 28:2037.

Mohamed Al-Badrashiny and Mona Diab. 2016. Lili: A Simple Language Independent Approach for Language Identification. In Proceedings of COLING 2016, the 26th International Conference on Computational Linguistics: Technical Papers, pages 12111219.

Mohamed Al-Badrashiny, Heba Elfardy, and Mona Diab. 2015. Aida2: A hybrid approach for token and sentence level dialect identification in arabic. In Proceedings of the Nineteenth Conference on Computational Natural Language Learning, pages 4251.

Brigitte Bigi, Armelle Brun, Jean-Paul Haton, Kamel Smaili, and Imed Zitouni. 2001. A comparative study of Topic Identification on Newspaper and Email. In Proceedings of the 8th International Symposium on String Processing and Information Retrieval - SPIRE'01, pages 238-241, Laguna de San Rafael, Chili.

Houda Bouamor, Nizar Habash, and Kemal Oflazer. 2014. A Multidialectal Parallel Corpus of Arabic. In Proceedings of the Language Resources and Evaluation Conference, LREC-2014, pages 1240 1245.

Houda Bouamor, Nizar Habash, Mohammad Salameh, Wajdi Zaghouani, Owen Rambow, Dana Abdulrahim, Ossama Obeid, Salam Khalifa, Fadhl Eryani,
Alexander Erdmann, and Kemal Oflazer. 2018. The MADAR Arabic Dialect Corpus and Lexicon. In Proceedings of the 11th International Conference on Language Resources and Evaluation, pages 3387 3396.

Houda Bouamor, Sabit Hassan, and Nizar Habash. 2019. The MADAR Shared Task on Arabic FineGrained Dialect Identification. In Proceedings of the Fourth Arabic Natural Language Processing Workshop (WANLP19), Florence, Italy.

Soumia Bougrine, Hadda Cherroun, and Djelloul Ziadi. 2015. Prosody-based spoken Algerian Arabic dialect identification. In International Conference on Natural Language and Speech Processing, ICNLSP'2015.

Ryan Cotterell and Chris Callison-Bursh. 2014. A multidialect, multi-genre corpus of informal written Arabic. In Proceedings of the Language Resources and Evaluation Conference, LREC-2014, pages 241-245.

Kareem Darwish, Hassan Sajjad, and Hamdy Mubarak. 2014. Verifiably effective Arabic dialect identification. In Proceedings of the EMNLP 2014 Workshop on Arabic Natural Language Processing (ANLP, pages 1465-1468, Doha, Qatar. Association for Computational Linguistic.

Heba Elfardy and Mona Diab. 2013. Sentence Level Dialect Identification in Arabic. In $A C L$ (2), pages 456-461.

Cyril Goutte, Serge LÉger, and Marine Carpuat. 2014. The nrc system for Discriminating Similar Languages. In Proceedings of the First Workshop on Applying NLP Tools to Similar Languages, Varieties and Dialects, pages 139-145.

Nizar Habash, Owen Rambow, Mona Diab, and Reem Kanjawi-Faraj. 2008. Guidelines for annotation of Arabic dialectness. In Proceedings of the Lrec workshop on hlt and nlp within the Arabic world, pages 49-53.

Salima Harrat, Karima Meftouh, and Kamel Smaïli. 2017. Machine translation for Arabic dialects (survey). Information Processing and Management.

Shervin Malmasi, Eshrag. Refaee, and Mark. Dras. 2015. Arabic dialect identification using a parallel multidialectal corpus. In In International Conference of the Pacific Association for Computational Linguistics, pages 35-53. Springer.

Andrew McCallum and Kamal Nigam. 1998. A comparison of event models for naive bayes text classification. In IN AAAI-98 WORKSHOP ON LEARNING FOR TEXT CATEGORIZATION, pages 41-48. AAAI Press.

Karima Meftouh, Salima Harrat, and Kamel Smaïli. 2018. PADIC: extension and new experiments. In 7th International Conference on Advanced Technologies ICAT, Antalya, Turkey. 
Tomas Mikolov, Kai Chen, Greg Corrado, and Jeffrey Dean. 2013. Efficient estimation of word representations in vector space. In ICLR (Workshop).

Fabian. Pedregosa, Gaël Varoquaux, Alexandre Gramfort, Vincent. Michel, Bertrand Thirion, Olivier Grisel, Mathieu Blondel, Peter Prettenhofer, Ron Weiss, Vincent Dubourg, Jake Vanderplas, Alexandre Passos, David Cournapeau, Matthieu Brucher, Matthieu Perrot, and Edouard Duchesnay. 2011. Scikit-learn: Machine learning in Python. Journal of Machine Learning Research, 12:2825-2830.

Toqir Rana, Yu-N Cheah, and Sukumar Letchmunan. 2016. Topic modeling in sentiment analysis: A systematic review. Journal of ICT Research and Applications, 10:76-93.

Motaz Saad, David Langlois, and Kamel Smaïli. 2013. Comparing Multilingual Comparable Articles Based On Opinions. In Proceedings of the 6th Workshop on Building and Using Comparable Corpora, pages 105-111, Sofia, Bulgaria. Association for Computational Linguistics ACL.

Fatiha Sadat, Farzindar Kazemi, and Atef Farzindar. 2014. Automatic identification of arabic dialects in social media. In In Proceedings of the first international workshop on Social media retrieval and analysis, page 3540. ACM.

Hasim Sak, Andrew Senior, and Franoise Beaufays. 2015. Long short-term memory recurrent neural network architectures for large scale acoustic modeling. In Google, USA.

Mohamed Salameh, Houda Bouamor, and Nizar Habash. 2018. Fine-grained Arabic Dialect Identification. In Proceedings of the 27th International Conference on Computational Linguistics, pages 1332-1344. Association for Computational Linguistics.

Karen Spärck Jones. 1972. A Statistical Interpretation of Term Specificity and Its Application in Retrieval. Journal of Documentation, 28:11-21.

Omar Zaidan and Chris Callison-Burch. 2012. Arabic Dialect Identification. Association for Computational Linguistics, Volume 1, pages 1-35. 